\title{
Nova Geminorum 1912 and the Origin of the Idea of Gravitational Lensing
}

\author{
Tilman Sauer \\ Einstein Papers Project \\ California Institute of Technology 20-7 \\ Pasadena, CA 91125, USA \\ tilman@einstein.caltech.edu
}

\begin{abstract}
Einstein's early calculations of gravitational lensing, contained in a scratch notebook and dated to the spring of 1912, are reexamined. A hitherto unknown letter by Einstein suggests that he entertained the idea of explaining the phenomenon of new stars by gravitational lensing in the fall of 1915 much more seriously than was previously assumed. A reexamination of the relevant calculations by Einstein shows that, indeed, at least some of them most likely date from early October 1915. But in support of earlier historical interpretation of Einstein's notes, it is argued that the appearance of Nova Geminorum 1912 (DN Gem) in March 1912 may, in fact, provide a relevant context and motivation for Einstein's lensing calculations on the occasion of his first meeting with Erwin Freundlich during a visit in Berlin in April 1912. We also comment on the significance of Einstein's consideration of gravitational lensing in the fall of 1915 for the reconstruction of Einstein's final steps in his path towards general relativity.
\end{abstract}

\section{Introduction}

Several years ago, it was discovered that Einstein had investigated the idea of geometric stellar lensing more than twenty years before the publication 
of his seminal note on the subject 1 The analysis of a scratch notebook 2 showed that he had derived equations in notes dated to the year 1912 that are equivalent to those that he would only publish in 1936.3 In the notes and in the paper, Einstein derived the basic lensing equation for a point-like light source and a point-like gravitating mass. From the lensing equation it follows readily that a terrestial observer will see a double image of a lensed star or, in the case of perfect alignment, a so-called "Einstein ring." Einstein also derived an expression for the apparent magnification of the light source as seen by a terrestial observer. The dating for the notes was based on other entries in the notebook. Some of these entries are related to a visit by Einstein in Berlin April 15-22, 1912, and it was conjectured that the occasion for the lensing entries was his meeting with the Berlin astronomer Erwin Freundlich during this week.

The lensing idea lay dormant with Einstein until in 1936 he was prodded by the amateur scientist Rudi W. Mandl into publishing his short note in Science. In the meantime, the idea surfaced occasionally in publications by other authors, such as Oliver Lodge (1919), Arthur Eddington (1920), and Orest Chwolson (1924) We only have one other piece of evidence that Einstein thought about the problem between 1912 and 1936. In a letter to his friend Heinrich Zangger, dated 8 or 15 October 1915, Einstein remarked that he has now convinced himself that the "new stars" have nothing to do with the lensing effect, and that with respect to the stellar populations in the sky the phenomenon would be far too rare to be observable 5

The Albert Einstein Archives in Jerusalem recently acquired a hitherto unknown letter by Einstein that both corroborates some of the historical conjectures of the early history of the lensing idea and also adds significant new insight into the context of Einstein's early considerations. From this letter it appears that the phenomenon of "new stars," i.e. the observation of this type of cataclysmic variables, played a much more prominent role in the origin of the idea than was suggested by the side remark in Einstein's letter to Zangger. It also adds important new information about Einstein's thinking in the crucial period between losing faith in the precursor theory to

\footnotetext{
${ }^{1}$ Renn, Sauer, and Stachel 1997] and Renn and Sauer 2003 .

2 Albert Einstein Archives (AEA), call number 3-013, published as CPAE3, Appendix A]. A facsimile is available on Einstein Archives Online at http://www.alberteinstein.info

${ }^{3}$ Einstein 1936.

${ }^{4}$ Lodge 1919, Eddington 1920, pp. 133-135], Chwolson 1924.

${ }^{5}$ Einstein to Heinrich Zangger, 8 or 15 October 1915 [CPAE8, Doc. 130].
} 
the general theory of relativity entertained in the years 1913-1915, and the breakthrough to a general relativistic theory of gravitation in the fall of 19156 In fact, the new letter justifies a reexamination of our reconstruction of what we know about Einstein's intellectual preoccupations both in April 1912 and in October 1915, and more generally about the genesis of the concept of gravitational lensing.

\section{Einstein's letter to Emil Budde}

The new letter is a response to Emil Arnold Budde (1842-1921), dated 22 May 1916.7 Budde had been director of the Charlottenburg works of the company of Siemens \& Halske from 1893 until 1911.8 He was the author of a number of scientific publications, among them a monograph on tensors in three-dimensional space [Budde 1914a] 9 and of a critical comment on relativity published in 1914 in the Verhandlungen of the German Physical Society 10

In an unknown letter to Einstein, Budde apparently had written about the possibility of observing what are now called Einstein rings, i.e. ring shaped images of a distant star that is in perfect alignment with a lensing star and a terrestial observer. The subject matter of Budde's initial letter can be inferred from Einstein's response in which he pointed out that one would expect the phenomenon to be extraordinarily rare, and that it could not be detected on photographic plates "as little circles" since irradiation would diffuse the images that would hence only appear as bright little discs, indistinguishable from the image of a regular star.

\footnotetext{
${ }^{6}$ For historical discussion, see [Norton 1984, Janssen et al. 2007, and further references cited therein.

${ }^{7}$ AEA 123-079. The letter will be published in the forthcoming volume of the Collected Papers of Albert Einstein.

${ }^{8}$ Budde had studied catholic theology and science, and had worked as a secondary school teacher and as a correspondent for the German daily Kölnische Zeitung in Paris, Rome, and Constantinople. In 1887, he became a Privatgelehrter in Berlin, edited the journal Fortschritte der Physik, and entered the company Siemens \& Halske as a physicist in 1892. In 1911, he retired and moved to Feldafing, near Lake Starnberg, since he had been advised by his physicians to live at an altitude of at least 600m Laue 1921, Werner 1921.

${ }^{9}$ In [Norton 1992, pp. 309-310] this textbook is cited as evidence for the argument that Grossmann's generalization of the term 'tensor' in Einstein and Grossmann 1913, was an original development.

${ }^{10}$ Budde 1914b], Budde 1914c].
} 
The interesting part of Einstein's response follows after this negative comment. Einstein continued to relate that he himself had put his hopes on a different aspect, namely that "due to the lensing effect" the distant star would appear with an "immensely increased intensity," and that he initially had thought that this would provide an explanation of the "new stars." He went on to list three reasons why he had given up this hope after more careful consideration. First, the temporal development of the intensity of a nova is asymmetric. The luminosity increases much faster than it declines again. Second, the color of the novae usually changes towards the red and, in general, its spectral character changes in a distinct and characteristic way. Third, the phenomenon would be very unlikely for the same reasons that the observation of an Einstein ring would be unlikely.

In the beginning of his letter, Einstein pointed out that Budde's idea concerned the same thing that "about half a year ago" ("vor etwa einem halben Jahre") had put him into "joyous excitement" ("freudige Aufregung"). At the end of the letter, he again wrote that the joy had been "just as short as it had been great." Counting back six months from the date of Einstein's letter, 22 May 1916, takes us to the 22nd of November 1915, which is just the time of the final formulation of general relativity. It is also just another six weeks or so away from the date of his letter to Zangger of early October, in which he wrote about the very same subject of the possible explanation of novae as a phenomenon of gravitational lensing.

\section{The lensing calculations in the scratch note- book}

In light of this new letter, let us briefly reexamine the calculations in the Scratch Notebook that had been dated to April 191211 Stellar gravitational lensing is an implicit consequence of a law of the deflection of light rays in a gravitational field. Such a law had been obtained by Einstein in 1911 as a direct consequence of the equivalence hypothesis. The angle of deflection

\footnotetext{
${ }^{11}$ The following brief recapitulation refers to [CPAE3, 585-586], or http://www.alberteinstein.info/db/ViewImage.do?DocumentID $=34432 \&$ Page $=23$ and $\cdots \&$ Page $=26$. For a complete and detailed paraphrase of Einstein's notes, see the Appendix below.
} 


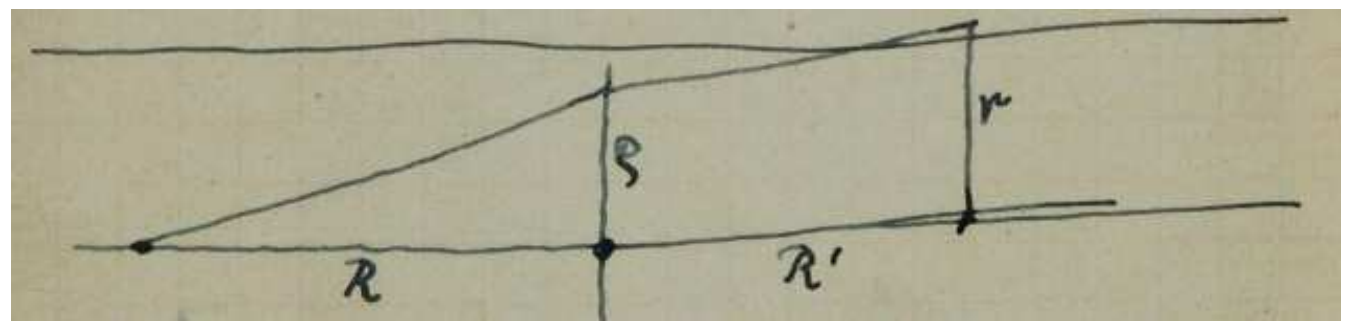

Figure 1: The geometric constellation for stellar gravitational lensing as sketched in Einstein's Scratch Notebook. From [CPAE3, p. 585].

â12 was found to be

$$
\tilde{\alpha}=\frac{2 k M}{c^{2} \Delta},
$$

where $k$ is the gravitational constant, $M$ the mass of the lensing star, $c$ the speed of light, and $\Delta$ the distance of closest approach of the light ray measured from the center of the massive star 13 On [p. 43] of the Scratch Notebook we find the sketch shown in Fig. (1) and underneath it the lensing equation

$$
r=\rho \frac{R+R^{\prime}}{R}-\frac{R^{\prime} \alpha}{\rho},
$$

where $R$ denotes the distance between the light emitting distant star and the massive star that is acting as a lens, $R^{\prime}$ the distance between the lensing star and the position of a terrestial observer who is located a distance $r$ away from the line connecting light source and lensing star. $\rho$ is the distance of closest approach of a light ray emitted by the star and seen by the observer. $\alpha=2 k M / c^{2}$ is a typical length (later known as the Schwarzschild radius) that depends on the mass of the light deflecting star and that determines

\footnotetext{
${ }^{12} \mathrm{I}$ am using the notation $\tilde{\alpha}$ instead of $\alpha$ (as in [Einstein 1911]) in order to distinguish this angle from the quantity $\alpha$ (effectively the Schwarzschild radius) in Einstein's scratch notebook.

${ }^{13}$ Einstein 1911, p. 908]. Qualitatively, Einstein had already derived the consequence of light bending in a gravitational field when he first formulated his equivalence hypothesis Einstein 1907, p. 461]. In the final theory of general relativity, the same relation is obtained with an additional factor of 2, as observed explicitly in [Einstein 1915c, p. 834]. Incidentally, the relevant formula was printed incorrectly by a factor of 2 in (the first printing of) Einstein's 1916 review paper of general relativity [Einstein 1916, p. 822], see [CPAE6, Doc. 30, n. 36] and also Einstein's response to Carl Runge, 8 November 1920 CPAE10, Doc. 195].
} 
the angle of deflection to be $\frac{\alpha}{\rho}$. The lensing equation can be written in dimensionless variables as

$$
r_{0}=\rho_{0}-\frac{1}{\rho_{0}}
$$

after defining $r_{0}$ and $\rho_{0}$ as

$$
\begin{aligned}
r_{0} & =r \sqrt{\frac{R}{R^{\prime}\left(R+R^{\prime}\right) \alpha}}, \\
\rho_{0} & =\rho \sqrt{\frac{R+R^{\prime}}{R R^{\prime} \alpha}} .
\end{aligned}
$$

The fact that equation (1) is a quadratic equation for $\rho_{0}$ entails that there are two solutions which correspond to two light rays that can reach an observer, along either side of the lensing star 14 and hence that a terrestial observer will see a double image of the distant star. For perfect alignment, the double image will turn into a ring shaped image, an "Einstein-ring" whose diameter $\rho_{0}^{\text {ring }}=\rho^{\text {ring }} \sqrt{\frac{R+R^{\prime}}{R R^{\prime} \alpha}}=1$ also follows immediately from the lensing equation.

In light of Einstein's letters to Zangger and Budde, it is interesting that Einstein went on to compute also the apparent magnification, obtaining the following expression:

$$
\mathcal{H}_{\text {tot }}=H\left\{\frac{1}{1-\frac{1}{\rho_{1}^{4}}}+\frac{1}{\frac{1}{\rho_{2}^{4}}-1}\right\} .
$$

Here $\mathcal{H}_{\text {tot }}$ is the total intensity received by the observer, and $H$ the intensity of the star light at distance $R . \rho_{1,2}$ denote the two roots of the quadratic equation (11). The term in brackets gives the relative brightness, reducing to 1 if no lensing takes place. Finally, some order of magnitude calculations on these pages showed that the probability of observing this effect would be given by the probability of having two stars within a solid angle that would cover $10^{-15}$ of the sky, which is highly improbable given that the number of known stars at the time was of the order of $10^{6} .15$

Equations that are entirely equivalent to these were published much later, in 1936, in Einstein's note to Science 16

\footnotetext{
${ }^{14}$ Since only three points are given, the problem is intrinsically a planar one, as long as the three points are not in perfect alignment.

${ }^{15}$ See the discussion in the appendix.

${ }^{16}$ Renn, Sauer, and Stachel 1997.
} 
The dating of the lensing notes in the scratch notebook to Einstein's visit in Berlin in April 1912 was based on other evidence in the notebook. Most importantly, p. [36] lists Einstein's appointments during his Berlin visit. In addition, pp. [38] and [39] recapitulate very specifically the equations of Einstein's two papers on the theory of the static gravitational field of February and March 1912, respectively 17 The calculations that deal with the lensing problem then appear on pp. [43]-[48], and on pp. [51] and [52] of the notebook. The sheet containing pp. [44] and [45] is a loose sheet inserted between p. [43] and p. [45]. After p. [53], three pages have been torn out, and then follow 37 blank pages, with some pages torn out in between. The remainder of the notebook contains entries that begin at the other end of the notebook which was turned upside down. Except for some apparently unrelated and undated entires on pp. [49], [50],18 and [54], the lensing calculations hence are at the end of a more or less continuous flow of entries. These physical characteristics of the notebook lead to an important consequence. All information that was pointing to a date of the lensing calculations in the year 1912 preceded the actual lensing calculations. Reexaming pp. [51] and [52] of the notebook in light of the letters to Zangger and to Budde in fact reveals that at least these entries were not written in 1912, but rather most likely at the time of the letter to Zangger, in early October 1915. There are two reasons for this. First, at the top of p. [51], Einstein wrote down the title of a book published only in 1914 19 Therefore, the following calculations are almost certainly to be dated later than the publication of this book. Second, at the bottom of p. [52], Einstein explicitly refers to the "apparent diameter of a Nova st[ar]." The calculations on pp. [51] and [52] in fact are a calculation of the apparent brightness and diameter of a star. We conclude that, in all probability, the calculations on pp. [51] and [52] were written at the time of Einstein's letter to Zangger, early October 1915.

Does the dating of pp. [51] and [52] to October 1915 also compel us to

\footnotetext{
${ }^{17}$ Einstein 1912a, Einstein 1912b].

${ }^{18}$ On the bottom half of p. [49] there is a sketch of Pascal's and Brianchon's Theorems, which deal with hexagons inscribed in or circumscribed on a conical section. I wish to thank Jesper Lützen for this identification. Other entries on pp. [49] and [50] also appear to deal with problems from projective geometry. There is also a sketch of a vessel filled with a liquid and the words "eau glyceriné" and what appears to be sketch of a magnetic moment in a sinusoidal magnetic field.

${ }^{19}$ Fernau 1914]. Could it be that the book was mentioned to Einstein when he met with Romain Rolland in Geneva in September 1915, see [CPAE8, Doc. 118]?
} 
revise our dating of the other lensing calculations in the notebook? To answer this question, we need to consider the broader historical context of the notes. But before doing so, we first observe that pp. [49] and [50] contain entries that appear unrelated to the lensing problem. As shown by the detailed paraphrase given in the appendix, the calculations on pp. [43] to [48] on the other hand represent a coherent train of thought, as do the calculations of pp. [51] and [52]. We also note that Einstein used a slightly different notation on pp. [43]ff. and on pp. [51]-[52]. In the first set, he denoted the distances between light source and lens and between lens and observer as $R$ and $R^{\prime}$, respectively. On pp. [51]-[52] he used the notation $R_{1}$ and $R_{2}$, respectively. He also reversed the roles of $r$ and $\rho$. We conclude that there is a discontinuity between the first set of lensing calculations on pp. [43] to [48] and the second set on pp. [51] and p. [52].

\section{The context of Einstein's early lensing cal- culations}

From Einstein's letter to Budde we learn that he had investigated the idea that stellar lensing might explain the phenomenon of the "new stars," and that he had given up this idea after looking more closely into the characteristic features of novae, especially their light curves and the changes in their spectral characteristics. Let us therefore briefly look into the astronomical knowledge about novae at the time.

The observation of a new star is an event that, in the early twentieth century, occurred only every few years. Between 1900 and 1915, eight novae were observed:20 Nova Persei 1901 (GK Per), Nova Geminorum (1) 1903 (DM Gem), Nova Aquilae 1905 (V604 Aql), Nova Vela 1905 (CN Vel), Nova Arae 1910 (OY Ara), Nova Lacertae 1910 (DI Lac), Nova Sagittarii 1910 (V999 Sgr), and Nova Geminorum (2) 1912 (DN Gem) with maximum brightness of $0.2,4.8,8.2,10.2,6.0,4.6,8.0,3.5$ magnitudes, respectively. At the time, "the two most interesting Novae of the present century," Campbell 1914, p. 493], were Nova Persei of 1901 and Nova Geminorum of 1912. The next spectacular nova to occur was the very bright Nova Aquilae 1918 (V603 Aql) with a maximum brightness of -1.1 mag.

Nova Geminorum (2) was discovered on March 12, 1912, by the as-

\footnotetext{
${ }^{20}$ For the following, see [Duerbeck 1987].
} 


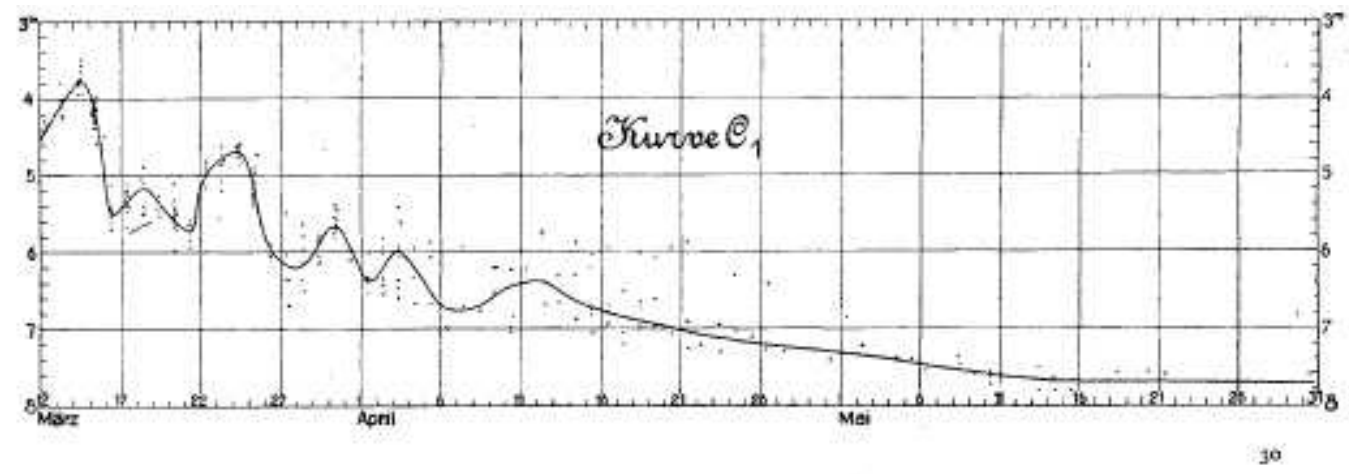

Figure 2: The light curve of Nova Geminorum 1912 for the first three months after its appearance, as put together by Fischer-Petersen on the basis of 253 individual observations. The points are the magnitudes reported by the individual observers, the solid line is to guide the eye. From [Fischer-Petersen 1912, p.429].

tronomer Sigurd Enebo at Dombaas, Norway Pickering 1912. On a photographic plate taken at Harvard College Observatory on March 10, showing stars of magnitude 10.5, it was not visible, but it was visible as a magnitude 5 star in the constellation Gemini on a Harvard plate of March 11. On March 13, a cablegram was received at Harvard and distributed throughout the United States. In the following days all major observatories as well as many amateur astronomers pointed their instruments towards the new star. The maximum brightness of mag 3.5 was reached on March 14 (Einstein's 33rd birthday!) Fischer-Petersen 1912. By March 16, the brightness was down to a magnitude of 5.5 and in the following weeks it decreased further, with distinct oscillations. By mid-April 1912, most observers registered a brightness of mag $6 \approx 7$, see Fig. (2). We now know that the DN Gem is a fast nova with a $t_{3}$-time of $37 \mathrm{~d}$. Its light curve is type $\mathrm{Bb}$ in the classification of [Duerbeck 1987], i.e. it declines with major fluctuations.

Like all classical novae, Nova Geminorum is, in fact, a binary system of a white dwarf and main sequence star, where hydrogen-rich matter is being accreted onto the white dwarf. Recent observations have even determined the binary period Retter et al. 1999]. The eruption of a classical nova occurs when a hydrogen-rich envelope of the white dwarf suffers a thermonuclear 
runaway 21 This explanation of classical novae also entails that they display the same sequence of spectral behaviour as the luminosity decreases, see also Fig. (3) below. However, our current understanding of classical novae was suggested only in the fifties, 22

The temporal proximity of the appearance of Nova Geminorum 1912 with Einstein's Berlin visit during the week of April 15-22, suggests that this astronomical event was discussed also when Einstein met with Freundlich for the first time 23 We know that the observatory in Potsdam took a number of photographs of the new star between March 15 and April 12 Furuhjelm 1912, Ludendorff 1912, and that Freundlich, among others, was charged with photometric observations of the nova [Fischer-Petersen 1912, p. 429]. Einstein and Freundlich had earlier corresponded about the possiblity of observing gravitational light deflection through the gravitational field of the sun 24 The purpose of their meeting was to discuss possible astronomical tests of Einstein's emerging relativistic theory of gravitation. The recent observation of the brightest nova since 1901 must have been on Freundlich's mind, and it seems more than likely that the idea of explaining the phenomenon in terms of gravitational lensing therefore came up in the course of their conversation. We conclude that our earlier dating of the first set of calculations of the lensing problem in the Scratch Notebook to the time of Einstein's encounter with Freundlich in April 1912 is the most likely possibility.

In fact, the context of the observation of Nova Geminorum 1912 provides an answer to the question as to why Einstein would have done the calculations at all and, in particular, why he would not have been content at the time with a calculation of the lensing equation, the separation of the double star image and, perhaps, the radius of the Einstein ring. Without this context it might seem a rather ingenious move on Einstein's part to go ahead and immediately compute the apparent magnification of the lensed star as well. But this answer to the question of motivation for the specific details of the

\footnotetext{
${ }^{21}$ For a review, see Shara 1989.

${ }^{22}$ For a historical overview of previous theories, see Duerbeck 2007.

${ }^{23}$ For evidence that Einstein met with Freundlich, see his letter to Michele Besso, 26 March 1912, in which he mentions planned discussions ("Besprechungen") with Nernst, Planck, Rubens, Warburg, Haber, and "an astronomer" - presumably Freundlich CPAE5, Doc. 377].

${ }^{24}$ Einstein to Freundlich, 1 September 1911, 21 September 1911, and 8 January 1912 CPAE5, Docs. 281, 287, 336].
} 


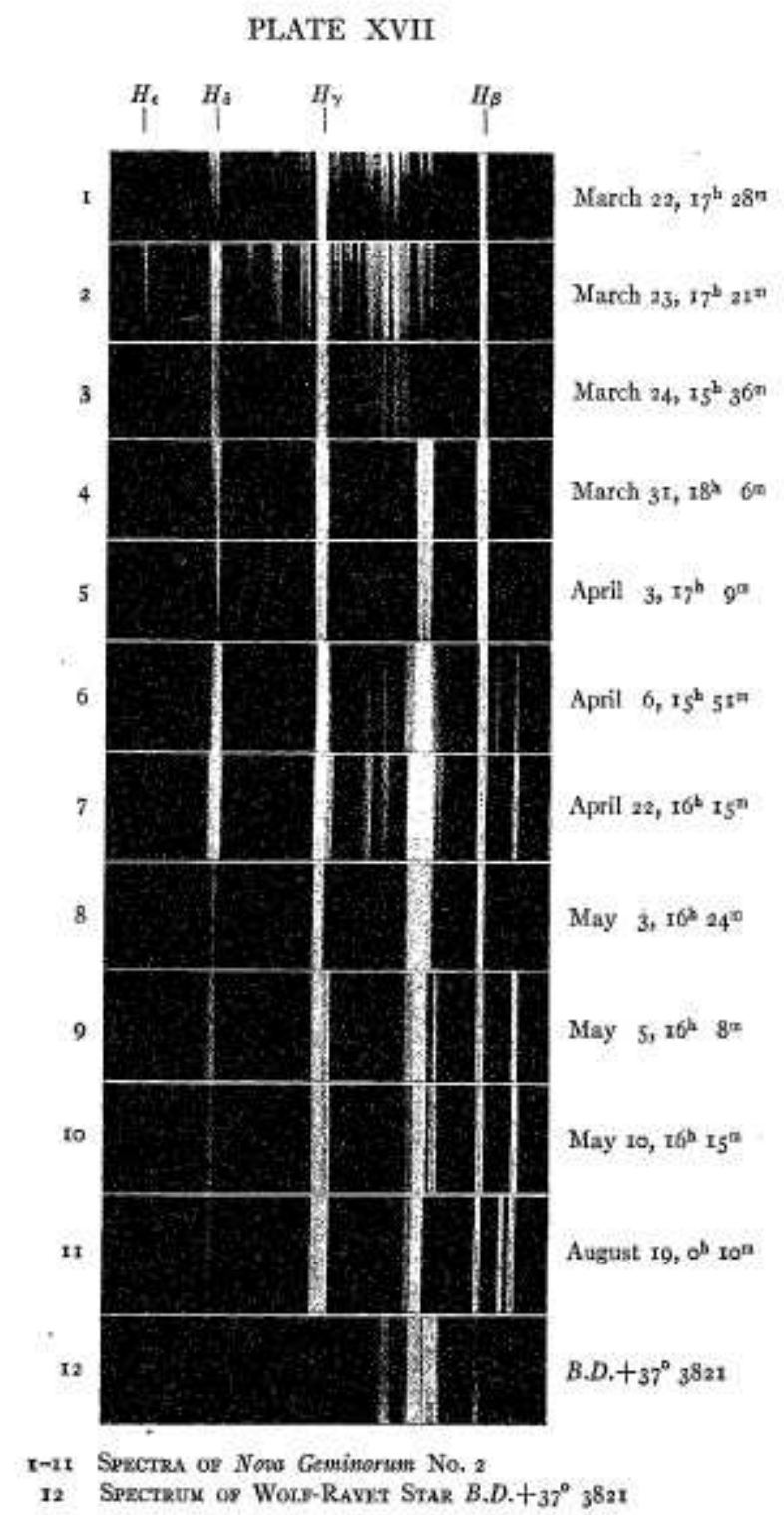

Figure 3: Changes in the spectrum of Nova Geminorum 1912, March 22 to August 19, 1912. From [Adams and Kohlschütter 1912. 
calculations in the Scratch Notebook, immediately raises another question.

Assuming that the first set of lensing calculations were done in spring 1912, why do we have no evidence that this idea was followed up by either Einstein or by Freundlich until the fall of 1915? To answer this question, it should first be observed that no summarizing results and analyses of the observations of Nova Geminorum 1912 were published before the end of the summer.

Let us briefly recall Einstein's intellectual preoccupations after his visit to Berlin in April 1912 25 Shortly before his trip to Berlin he had submitted his two papers on a theory of the static gravitational field 26 After his return to Prague in April 1912, Einstein was preparing for his move to Zurich. The two papers were published in the 23 May issue of the Annalen der Physik. Einstein wrote an addendum at proof stage to the second one, in which he showed that the equations of motion could be written in a variational form, adding that this would give us "an idea about how the equations of motion of the material point in a dynamic gravitational field are constructed" [Einstein 1912b, p. 458]. He also entered into a published dispute with Max Abraham on their respective theories of gravitation 27 At the end of July, he departed Prague for Zurich. The next thing we know about his work on gravitation comes from a letter to Ludwig Hopf, dated 16 August 1912, in which he wrote:

The work on gravitation is going splendidly. Unless I am completely wrong, I have now found the most general equations 28

These most general equations are, in all probability, equations of motion in a gravitational field, represented by a metric tensor. After his arrival in Zurich, Einstein began a collaboration with his former classmate Marcel Grossmann, now his colleague at the ETH. Their research on a generalized

\footnotetext{
${ }^{25}$ We will focus here on his work of gravitation yet for the sake of completeness it should be noted that Einstein at the same time was also thinking about quantum theory, most notably about the law of photochemical equivalence and about the problem of zero point energy, see [CPAE4, Docs. 5, 6, 11, 12].

${ }^{26}$ Einstein 1912a, Einstein 1912b, were received by the Annalen der Physik on 26 February and 23 March, respectively.

${ }^{27}$ Einstein 1912C which was received by the Annalen on 4 July 1912 is a response to a critique by Abraham.

${ }^{28}$ Einstein to Hopf, 16 August 1912 [CPAE5, Doc. 416].
} 
theory of relativity is documented in Einstein's so-called "Zurich Notebook" 29 and culminates in the publication of the "Outline [Entwurf] of a generalized theory of relativity and a theory of gravitation," in early summer of 1913 coauthored with Marcel Grossmann 30 This so-called Entwurf-theory contains all the elements of the final theory of general relativity, except for generally relativistic field equations. Einstein would hold onto this theory until his final breakthrough to general relativity in the fall of 1915.

In conclusion, we observe that Einstein's path toward the general theory of relativity in 1912 took him deep into the unknown land of the mathematics associated with the metric tensor, before there was a chance to reconsider the lensing idea in light of the data for Nova Geminorum 1912. In any case, he would have to rely on Freundlich or other professional astronomers for a secure assessment of the possibilities of an observation of the lensing effect at the time.

Freundlich, on the other hand, continued to think about ways to test Einstein's new theory of gravitation 31 But his focus was on observations of light deflection during a solar eclipse 32 In August 1914, he led a first (unsuccessful) expedition to the Crimea to observe the eclipse of 21 August 1914. Even these efforts were hampered by the lack of funding and, more generally, by the difficulties of securing increased research time that would have allowed Freundlich to freely pursue his collaboration with Einstein.

Given these circumstances, and the fact that order-of-magnitude calculations may have convinced Einstein already in 1912 that the phenomenon would be rare, it seems plausible that the lensing idea was not pursued further for some time after Einstein's visit in Berlin in April 1912.

Let us finally reexamine the events of fall 1915. Einstein, in the meantime had left Zurich in the spring of 1914, accepting an appointment as member of the Prussian Academy in Berlin. In September 1915, Einstein spent a few weeks in Switzerland where he met, among others, with Heinrich Zangger, Michele Besso, and Romain Rolland. On 22 September 1915, he left Zurich 33 but travelled via Eisenach where he was on the 24th of September 34 By the

\footnotetext{
${ }^{29}$ AEA 3-006, see [CPAE4, Doc. 10]. For a comprehensive discussion of this document, including a facsimile, transcription, and detailed paraphrase, see Janssen et al. 2007.

30 Einstein and Grossmann 1913 .

${ }^{31}$ See Hentschel 1994 and Hentschel 1997.

${ }^{32} \mathrm{See}$ his correspondence with Einstein in CPAE5.

${ }^{33}$ CPAE8, p. 998].

34 CPAE10, Doc. Vol. 8, 122a].
} 
30th of September, at the latest, he was back in Berlin, and wrote a letter to Freundlich:

I am writing you now about a scientific matter that electrifies me enormously 35

It is clear from the letter, however, that the excitement indicated to Freundlich is not about the idea of gravitational lensing. Rather, Einstein had found an internal contradiction in his Entwurf theory that amounted to the realization that Minkowski space-time in rotating Cartesian coordinates would not be a solution of the Entwurf field equations 36 This insight undermined his confidence in the validity of the Entwurf theory, and is later mentioned as one of three arguments that induced Einstein to lose faith in the Entwurf equations 37 The first of these arguments was the fact that a calculation of the planetary perihelion advance in the framework of the Entwurf theory did not produce the well-known anomaly that had been established for Mercury. This problem had been known to Einstein for some time 38 The third argument was realized sometime in early October, a few days after stumbling upon the problem with rotation, and concerned the mathematical derivation of the Entwurf field equations in Einstein's comprehensive review of October 1914 In any case, we know that Einstein asked Freundlich to look into the problem of the rotating metric, and that they met some time in early October. This follows from a letter Einstein wrote to Otto Naumann,

\footnotetext{
${ }^{35}$ Einstein to Freundlich, 30 September 1915 [CPAE8, Doc. 123]. For a detailed discussion of this letter and its significance for the reconstruction of Einstein's final breakthrough to general relativity, see Janssen 1999.

${ }^{36}$ Interestingly, the Scratch Notebook contains an entry that is pertinent to this problem. On p. [66], i.e. on the last page of the backward end of the notebook, Einstein considers the case of rotation in a calculation that exactly matches corresponding calculations dating from October 1915, see Janssen 1999. Janssen cautiously remarks that he believes this calculation to date from 1913 [Janssen 1999, p. 139]. It seems possible, however, that these entries as well as the immediately preceding ones on the perihelion advance (see note 38) may well date from late 1915 as well.

${ }^{37}$ See Einstein to Arnold Sommerfeld, 28 November 1915, and to Hendrik A. Lorentz, 1 January 1916 [CPAE8, Docs. 153, 177].

${ }^{38}$ See Earman and Janssen 1993 and [CPAE4, pp. 344-359]. The Scratch Notebook contains some calculations related to the perihelion advance on pp. [61-66], i.e. in the backward end of the notebook. On p. [61], Einstein there explicitly noted that the advance of Mercury's perihelion would be $17^{\prime \prime}$ which is the value that is obtained on the basis of the Entwurf-theory. These calculations are undated, see note 36.

39 Einstein 1914.
} 
dated after 1 October 1915, in which Einstein asked about possibilities to allow Freundlich more freedom to pursue independent research. In this letter, Einstein mentioned that Freundlich had visited him "recently." 40

By 12 October, Einstein had realized the third problem with the Entwurf theory, the unproven uniqueness of the Lagrangian for the Entwurf field equations, as he reported in a letter to Lorentz. In this letter, he neither mentioned the problem with the rotating metric nor the issue of gravitational lensing 41

For our reconstruction of this episode, the precise date of Einstein's letter to Zangger in which he remarked that he had given up the hope of explaining the "new stars" as a lensing phenomenon is relevant. It could have been written either on the 8 th or the 15 th of October 42

The letter to Zangger suggests that they had talked about the idea earlier since Einstein seems to presuppose that Zangger knew what he was talking about and did not explain what he meant by "lens effect" ("Linsenwirkung"). As mentioned before, Einstein had just recently met with Zangger, as well as with Besso before returning to Berlin. The following scenario seems therefore plausible:

Upon returning to Berlin some time after the 24th of September 1915, Einstein realized the problem of the rotating metric solution and wrote to Freundlich on the 30th, asking him to look into this issue. Shortly afterwards, the two met in person. Most likely they discussed not only the rotation problem, but also the lensing idea. Having found troubling indications of an inner inconsistency in the very foundations of this theory, it would have been a natural move for Einstein to go back and reconsider early arguments such as one based safely on the equivalence hypothesis 43 After this meeting, Einstein

\footnotetext{
40 "Letzter Tage war Herr Dr. Freundlich von der Sternwarte N bei mir." CPAE8, Doc. 124].

${ }^{41}$ In a letter to Hilbert, dated 7 November 1915, Einstein wrote that he realized the flaw in his proof "about four weeks ago" [CPAE8, Doc. 136].

${ }^{42}$ The editors of [CPAE8] dated this letter explicitly to the 15th of October. It seems, however, that the 8 th is also a possibility. The letter was written on a Friday between September 30, when a fire and explosion took place in the comb factory Walter near Lake Biel took place, mentioned in the letter, and October 22 when Einstein participated in the first Academy session after the summer break. I see no reason why Einstein could not have heard of the accidents from Zangger before October 8.

${ }^{43}$ It seems unlikely that Einstein at that time was already contemplating a quantitatively different law of light deflection. Einstein first observed in [Einstein 1915c, p. 834] that an additional factor of 2 would arise from the different first-order approximation for the
} 
wrote to Naumann exploring possibilities to give Freundlich more research freedom. By October 8, Einstein had convinced himself that gravitational lensing cannot explain the "new stars." On 12 October, he realized the third problem of his mathematical derivation of the Entwurf field equation.

According to this reconstruction of the sequence of events, it is remarkable that the "joyous excitement" about the lensing idea falls within days after his being "electrified" about the realization of the rotation problem on 30 September, and his realization of the third problem of the mathematical derivation of the Entwurf equation, on or before 12 October 1915.44

Some five weeks later, his excitement was even greater and his heart, allegedly, skipped a beat when he found that he could derive the anomalous advance of Mercury's perihelion on the basis of his new field equations. And after having submitted the last of his four November communications to the Prussian Academy on 25 November which presented the final gravitational field equations, the "Einstein equations," he wrote to Sommerfeld:

You must not be cross with me that I am answering your kind and interesting letter only today. But in the last month I had one of the most exciting, exhausting times of my life, indeed also one of the most successful. I could not think of writing 45

It is interesting to learn from Einstein's letter to Budde that in addition to the realization of the problems with the Entwurf theory and the eventual suc-

metric if the Newtonian limit is derived on the basis of generally covariant field equations in which the Ricci tensor is directly set proportional to the energy-momentum tensor. These latter equations were published in his second November memoir, presented on 11 November, under the assumption that the trace of the energy-momentum tensor vanish. In his comment on the factor of 2, Einstein refers to this result as being in contrast to "earlier calculations" where the hypothesis of vanishing energy-momentum had not yet been made.

${ }^{44}$ For completeness, one should point one other intellectual activity of Einstein's during those days. In Einstein's letter to Zangger of 8 or 15 October, he also mentioned that he wrote "a supplementary paper to my last year's analysis on general relativity." The last year's analysis is, in all likelyhood [Einstein 1914]; the supplementary paper is, in all likelihood, an early version of Einstein 1916b, or, perhaps, an early version of Einstein's first November memoir [Einstein 1915a, see [CPAE8, Doc. 130, note 5] and Janssen 1999, note 51].

45 "Sie dürfen mir nicht böse sein, dass ich erst heute auf Ihren freundlichen und interessanten Brief antworte. Aber ich hatte im letzten Monat eine der aufregendsten, anstrengendsten Zeiten meines Lebens, allerdings auch der erfolgreichsten." Einstein to Sommerfeld, 28 November 1915 [CPAE8, Doc. 153]. 
cess of his breakthrough to general relativity, an astronomical problem, the idea of explaining novae in terms of gravitational lensing added to Einstein's excitement in the midst of what must indeed have been the most intense period of intellectual turmoil in his life.

\section{Concluding remarks}

Einstein's recollections of his thought concerning the explanation of the "new stars" as a phenomenon of gravitational lensing in his letter to Budde add two significant insights to our reconstruction of the genesis of general relativity. If our dating and context hypothesis of the lensing calculations in the scratch notebook are correct, we learn that it was an astronomical observation that triggered the elaboration of a significant consequence of the equivalence hypothesis and its consequence of gravitational light deflection. It is also interesting that on his intellectual path from the Entwurf theory to the final theory of general relativity, Einstein also took a detour in which he explored further consequences of one of the solid pillars of general relativity, the equivalence hypothesis.

\section{Appendix: Einstein's lensing calculations in the Scratch Notebook AEA 3-013}

The following is a self-contained line-by-line paraphrase of Einstein's lensing calculations in his scratch notebook, CPAE3, pp. 585-589]. The pagination in square brackets refers to the sequence of pages in the notebook.

The calculations start out on p. [43] with Fig. (1) and continue on the facing page p. [46]. From the more explicit sketch in Fig. (44), we read off the lensing equation:

$$
r=\rho \frac{R+R^{\prime}}{R}-\frac{R^{\prime} \alpha}{\rho} .
$$

Here $R$ is the distance between the light emitting star $S$ and the lensing star $L ; R^{\prime}$ the distance between the massive star $L$ and the projected position of the observer $O$ on the line connecting light source and lens; $\rho$ is the distance of closest approach of a light ray emitted from the distant star and seen by an observer; $r$ is the orthogonal distance of the terrestial observer to the line connecting light source and lens. The first term in the lensing equation 


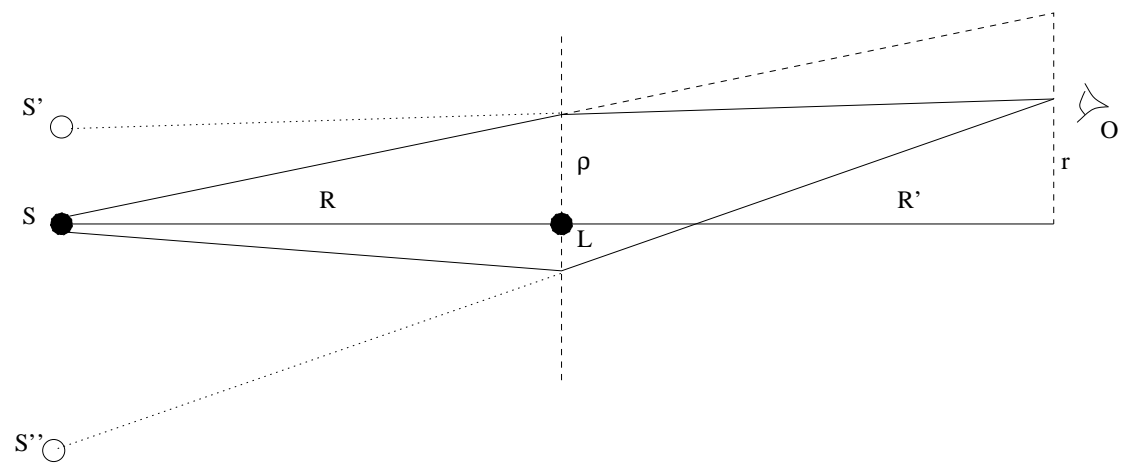

Figure 4: The geometry of stellar lensing.

(44) is obtained from the similarity of triangles with baseline $R$ and $R+R^{\prime}$, respectively, and the second term is the angle of deflection as given by the law of gravitational light bending, where $\alpha$ is the Schwarzschild radius $2 G M / c^{2}$. If we want to write this equation in dimensionless variables, we need to multiply it by a factor of

$$
\sqrt{\frac{R}{R^{\prime}\left(R+R^{\prime}\right) \alpha}}
$$

so that, when we define $r_{0}$ and $\rho_{0}$ as

$$
\begin{aligned}
& r_{0}=r \sqrt{\frac{R}{R^{\prime}\left(R+R^{\prime}\right) \alpha}} \\
& \rho_{0}=\rho \sqrt{\frac{R+R^{\prime}}{R R^{\prime} \alpha}}
\end{aligned}
$$

the lensing equation (44) turns into

$$
r_{0}=\rho_{0}-\frac{1}{\rho_{0}} .
$$

This is a quadratic equation for $r_{0}$, the two solutions of which correspond to the two light rays passing above and below $L$. The observer $O$ therefore sees two images of $S$ at positions $S^{\prime}$ and $S^{\prime \prime}$, respectively. To read off the radius of an "Einstein ring," obtained for perfect alignment of $S, L$, and $O$, one only needs to set $r_{0} \equiv 1$. 
In order to get an expression for the apparent magnification, Einstein proceeded as follows. He first took the square of eq. (8) as

$$
2+r^{2}=\rho^{2}+\frac{1}{\rho^{2}}
$$

If we multiply this equation by $\pi$ and denote the areas of the circles corresponding to the radii $r$ and $\rho$ as $f=\pi r^{2}$ and $\varphi=\pi \rho^{2}$, respectively, we can write this equation as

$$
2 \pi+f=\varphi+\frac{\pi^{2}}{\varphi}
$$

We are not interested in the full circle corresponding to these radii but in the differential area element associated with these radii. More precisely, we are interested in the change of the differential area element $d f$ associated with $f$ when we change the differential area element $d \varphi$ associated with $\varphi$. Hence, Einstein wrote

$$
d f=\left(1-\frac{\pi^{2}}{\varphi^{2}}\right) d \varphi=\left(1-\frac{1}{\rho^{4}}\right) d \varphi .
$$

The intensity $\mathcal{H}$ of the brightness received at $r$ is related to the intensity $H$ of the brightness at $\rho$ by

$$
\mathcal{H} d f= \pm H d \varphi
$$

where the plus and minus signs refer to the two solutions of the quadratic equation. Since we have from (11)

$$
\frac{d f}{d \varphi}=\left(1-\frac{1}{\rho^{4}}\right)
$$

we get

$$
\mathcal{H}= \pm \frac{H}{1-\frac{1}{\rho^{4}}} .
$$

or, inserting the explicit solutions, we can write the total brightness at $r$ as

$$
\mathcal{H}_{\text {tot }}=H\left\{\frac{1}{1-\frac{1}{\rho_{1}^{4}}}+\frac{1}{\frac{1}{\rho_{2}^{4}}-1}\right\} .
$$

As Einstein remarked, the term in brackets gives the relative brightness, if we take the value for $r \rightarrow \infty$ to be 146 This result is equation number (3)

\footnotetext{
46 "Klammer gibt relative Helligkeit"
} 
in Einstein's notes, and most of the following material on pp. [47] and [48], as well as on the loose sheet containing pp. [44] and [45], will be a discussion of this expression for the relative brightness.

On p. [47], Einstein first rewrote the reduced lensing equation as

$$
r=\frac{1}{x}-x
$$

and then the terms in brackets as

$$
\{\}=\frac{1}{1-x_{1}^{4}}+\frac{1}{x_{2}^{4}-1} .
$$

The next step is to bring the two terms to a common denominator 47

$$
\mathcal{H}_{r}=\frac{x_{1}^{4}-x_{2}^{4}}{\left(1-x_{1}^{4}\right)\left(1-x_{2}^{4}\right)} .
$$

If one squares the lensing equation (16) twice, one obtains

$$
-2+\left(2+r^{2}\right)^{2}=\frac{1}{x^{4}}+x^{4}
$$

If we now introduce new variables $A$ and $u$ via

$$
2 A=-2+\left(2+r^{2}\right)^{2}
$$

or

$$
A=-1+\frac{1}{2}\left(2+r^{2}\right)^{2}=1+2 r^{2}+\frac{1}{2} r^{4},
$$

and

$$
u=x^{4},
$$

we can write the quadrupled equation (19) as

$$
2 A=u+\frac{1}{u}
$$

Multiplication by $u$ and adding $A^{2}$ on each side gives

$$
u^{2}-2 A u+A^{2}=-1+A^{2},
$$

\footnotetext{
${ }^{47}$ In the notes, Einstein refers to this step as "Rationalisierung".
} 
from which one can immediately read off the two solutions of eq. (23) as

$$
u=-A \pm \sqrt{A^{2}-1}
$$

Given (18), the difference between the two roots,

$$
u_{1}-u_{2}=2 \sqrt{A^{2}-1}
$$

provides an expression for the nominator of $\mathcal{H}_{r}$ in (18). With the two roots, we can also rewrite the quadratic equation in the form

$$
u^{2}-2 A u+1=\left(u-u_{1}\right)\left(u-u_{2}\right)
$$

and if we now set $u=1$, we obtain

$$
2(1-A)=\left(1-u_{1}\right)\left(1-u_{2}\right)
$$

which gives us an expression for the denominator of $\mathcal{H}_{r}$ in (18). Combining the two expressions, as Einstein did on p. [48], we obtain

$$
\begin{aligned}
\mathcal{H}_{r} & =\sqrt{\frac{A+1}{A-1}} \\
& =\sqrt{1+\frac{1}{r^{2}\left(1+\frac{1}{4} r^{2}\right)}},
\end{aligned}
$$

where we have inserted (21) to obtain the second line.

We now have an explicit expression for the relative brightness as a function of the dimensionless variable $r$. We now evidently see that $\mathcal{H}_{r} \rightarrow 1 / r$ for $r \rightarrow 0$, and that $\mathcal{H}_{r}$ approaches 1 asymptotically from above for large $r$, see Fig. (15).

Let us now reconstruct Einstein's order-of-magnitude estimate for the expected frequency of the phenomenon on p. [45]. The explicit expression for the relative brightness gives us a measure of the maximal distance $r$ for which significant magnification is obtained. We can look at specific values of $\mathcal{H}_{\mathrm{r}}(r)$. For instance, for $r_{0}=\frac{1}{2}$ we find

$$
\mathcal{H}_{r}\left(\frac{1}{2}\right)=\sqrt{1+\frac{1}{\frac{1}{4}\left(1+\frac{1}{16}\right)}} \approx \sqrt{5} \approx 2 .
$$




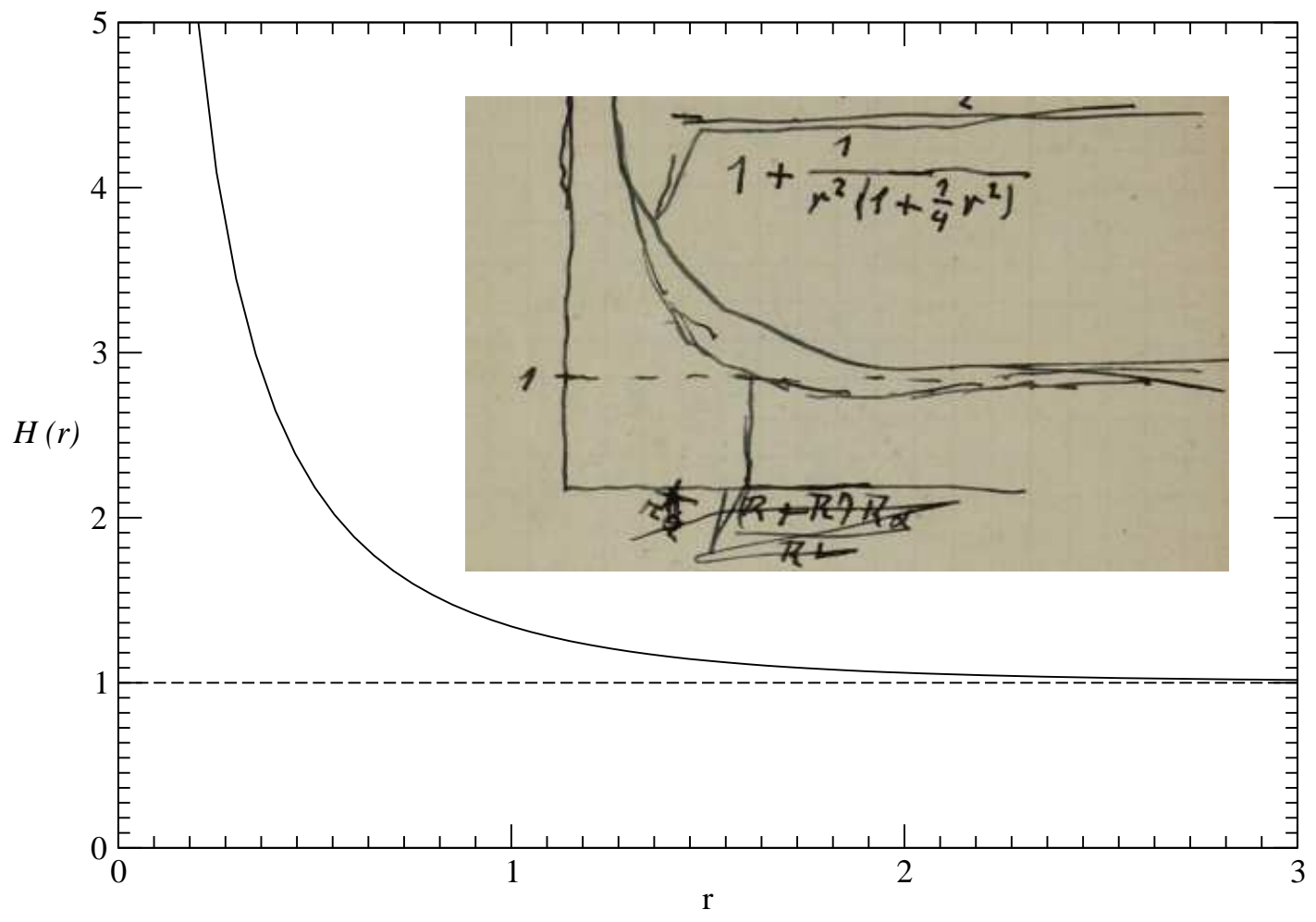

Figure 5: A plot of the expression (30) for the relative brightness $\mathcal{H}_{\mathrm{r}}$ as a function of $r$. The inset is from [CPAE3, p. 587]. 
Hence, Einstein concluded that up to a distance of $r_{0}=\frac{1}{2}$ one would obtain an increase of the intensity by a factor of 2 . In other words, if we write the intensity $\mathcal{H}_{\mathrm{r}_{0}}$ asymptotically for small $r_{0}$ and $R^{\prime} \gg R$ as

$$
\begin{aligned}
\mathcal{I}_{0} \frac{1}{r_{0}} & =\mathcal{I}_{0} \frac{1}{r} \sqrt{\frac{R^{\prime}\left(R+R^{\prime}\right) \alpha}{R}} \\
& \approx \mathcal{I}_{0} \frac{R^{\prime}}{r} \sqrt{\frac{\alpha}{R}},
\end{aligned}
$$

we see that for a lensing star at a distance of $R$, the relative increase in intensity is given by

$$
\frac{r}{R^{\prime}}=\operatorname{tg} \bar{\alpha}
$$

Here $\bar{\alpha}$ is the angle that determines how well the distant star has to be aligned with the lensing star and the observer to produce appreciable magnification. In order to get an order-of-magnitude estimate for this angle, one needs an order-of-magnitude estimate for $\sqrt{\frac{\alpha}{R}}$. In order to obtain such an estimate, Einstein notes that the ratio of the solar Schwarschild radius $\alpha$ to the solar equatorial radius $R_{s}$ is given approximately by

$$
\frac{\alpha}{R_{s}}=3 \cdot 10^{-6}
$$

The radius of the sun is 2 light seconds, and the distance of the nearest stars is of the order of 10 light years, or

$$
10^{5} \cdot 365 \cdot 10 \approx 4 \cdot 10^{8} \quad \text { lightseconds. }
$$

It follows that $\frac{\alpha}{R}$ for a star of 1 solar mass 10 lightyears away is

$$
\frac{\alpha}{R}=\frac{\alpha}{R_{s}} \cdot \frac{R_{s}}{R} \approx 10^{-14} \text { or } \quad \sqrt{\frac{\alpha}{R}} \approx 10^{-7} .
$$

To see the distant star with double intensity, we therefore have

$$
2=\frac{10^{-7}}{\operatorname{tg} \bar{\alpha}}
$$

so that the angle $\bar{\alpha}$ is of order $10^{-7}$. A linear angle corresponds to a solid angle roughly by taking its square. Thus, the angular size of the region where the distant star needs to be found behind a massive star in order to 
be magnified in the lens is of order $10^{-14}$. In angular units, the total sky has an area of $4 \pi \approx 10$, so that the angular size of the region in question covers a fraction of $10^{-15}$ of the total sky. This has to be contrasted with the average density of stellar population in the sky. The Bonner Durchmusterung listed of the order of $3 \cdot 10^{5}$ stars to ninth magnitude for the northern hemisphere, so a reasonable average density of the number of stars would be 1 star per $10^{-5}$ of the sky 48

On the back of the loose sheet [p. 44] we find a few more calculations related to order-of-magnitude estimates that start from (32). Einstein here again goes back to the definition of $r_{0}$ and $\rho_{0}$ in terms of $R, R^{\prime}$, and $\alpha 49$ Again, he observes that $r_{0}=\frac{1}{2}$ would give twice the usual intensity, and rewrites (6) for this case:

$$
r=\frac{1}{2} \sqrt{\frac{R^{\prime}\left(R+R^{\prime}\right) \alpha}{R} .}
$$

The latter equation for $R^{\prime} \gg R$ turns into

$$
\frac{r}{R^{\prime}} \approx \frac{1}{\sqrt{\alpha} R}
$$

and for $R \ll R^{\prime}$ into

$$
\frac{r}{R^{\prime}} \approx \frac{1}{2} \sqrt{\alpha} R^{\prime}
$$

Einstein concluded that the smaller of the two distances $R$ and $R^{\prime}$ determines the angle $\frac{r}{R^{\prime}}$. In the top right corner of the page, Einstein jotted down another order-of-magnitude calculation, which I do not fully understand. Apparently, he computed the distance of 100 lightyears in terms of centimeters

$$
3 \cdot 10^{10} \cdot 3 \cdot 10^{7} \cdot 10^{2} \quad[\mathrm{~cm}] \approx 10^{20} \mathrm{~cm}
$$

\footnotetext{
${ }^{48}$ On the relevant page under discussion here, we also find a little sketch by Einstein of a circle and the angle of its radius for a point some distance away. The precise meaning of this sketch is unclear but the numbers written next to it suggest that Einstein was considering the order of magnitude for the angular size of the moon. The radius of the moon is seen under an angle of $15^{\prime}$ from the earth, and the mean distance between the earth and the moon in units of the lunar radius is about 200, which translates to an angle of $50^{\circ}$.

${ }^{49}$ One can see here that Einstein corrected an error in his earlier calculations on [p. 43], where he had erroneously written the second term of the lensing equation (44) with $R$ instead of $R^{\prime}$, which resulted in a confusion of the factors of $R$ and $R^{\prime}$ in expressions (5) and (6).
} 


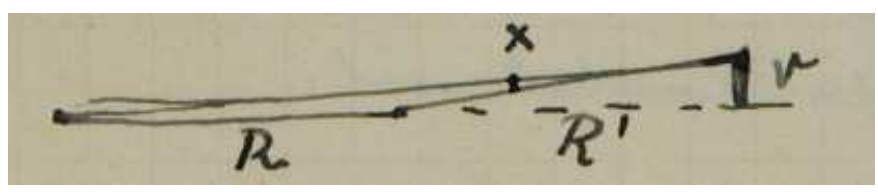

Figure 6: A sketch in Einstein's scratch notebook to obtain eq. (43). From [CPAE3, p.585].

He also computed the angle $x$ under which the star at distance $R^{\prime}$ and the star at distance $R+R^{\prime}$ would be seen by an observer at distance $r$ away from the connecting line between the two stars if no lensing took place:

$$
x=r\left\{\frac{1}{R^{\prime}}-\frac{1}{R+R^{\prime}}\right\}=r \frac{R}{R^{\prime}\left(R+R^{\prime}\right)} .
$$

The first equation can be read off from a little sketch of the geometry of light source, lensing star, and observer, at the bottom of the page, see Fig. (66).

Let us finally comment on the calculations on pp. [51] and [52]. As mentioned in the main text of this article, Einstein here introduced a change of notation. On p. [51], he sketched again the geometry for stellar lensing. Here, the geometry has been turned by 90 degrees, and the notation changed so that $R$ and $R^{\prime}$ become $R_{1}$ and $R_{2}$, and $\rho$ and $r$ are interchanged to become $r$ and $\rho$, respectively. This change of notation is reflected in the lensing equation, written down on p. [52] as

$$
\rho=r+R_{1}\left(w-\frac{\alpha}{r}\right)=\left(1+\frac{R_{1}}{R_{2}}\right) r-\frac{R_{2} \alpha}{r},
$$

where $\tan w=r / R_{2}$. Einstein then immediately proceeded to compute the magnification by taking the square of the lensing equation and then computing the derivative as

$$
\frac{d\left(\rho^{2}\right)}{d\left(r^{2}\right)}=\left(1+\frac{R_{1}}{R_{2}}\right)^{2}-\frac{\left(R_{1} \alpha\right)^{2}}{r^{4}}=\mathcal{A} \cdot \frac{H}{H_{0}} .
$$

Instead of pursueing this calculation further, Einstein instead wrote "apparent diameter of a Nova star," and wrote down the solution of eq. (44) for $\rho=0$, as to obtain the diameter of an Einstein ring:

$$
r_{0}=\sqrt{\frac{R_{1} R_{2} \alpha}{R_{1}+R_{2}}}
$$


He computed the angle $w_{0}$ as

$$
w_{0}=\sqrt{\frac{R_{1} \alpha}{R_{2}\left(R_{1}+R_{2}\right)}} .
$$

The calculation ends with an attempt at a numerical order-of-magnitude estimation which seems to proceed along the same lines as in eqs. (35, 36). The calculation, however, was broken off, and the whole page was struck through.

\section{Acknowledgments}

I wish to thank Diana Buchwald for a critical reading of an earlier version of this paper, and Hilmar Duerbeck for some helpful comments. Unpublished correspondence in the Albert Einstein Archives is quoted by kind permission.

\section{References}

[Adams and Kohlschütter 1912] Adams, Walter S., and Kohlschutter [sic], Arnold. "Observations of the spectrum of Nova Geminorum No. 2." Astrophysical Journal 36 (1912), 293-321.

[Budde 1914a] Budde, Emil Arnold. Tensoren und Dyaden im dreidimensionalen Raum. Braunschweig: Vieweg, 1914.

[Budde 1914b] Budde, Emil Arnold. "Kritisches zum Relativitätsprinzip." Verhandlungen der Deutschen Physikalischen Gesellschaft 16 (1914) 586-612.

[Budde 1914c] Budde, Emil Arnold. "Kritisches zum Relativitätsprinzip II." Verhandlungen der Deutschen Physikalischen Gesellschaft 16 (1914) 914-925.

[Campbell 1914] Campbell, Leon. "A systematic search for bright Novae." Popular Astronomy 22 (1914), 493-495.

[Chwolson 1924] Chwolson, Orest. "Über eine mögliche Form fiktiver Doppelsterne." Astronomische Nachrichten 221 (1924) 329-330. 
[CPAE2] Stachel, John, et al. (eds.) The Collected Papers of Albert Einstein, Vol. 2: The Swiss Years: Writings, 1900-1909, Princeton: Princeton University Press, 1989.

[CPAE3] Klein, Martin, et al. (eds.) The Collected Papers of Albert Einstein, Vol. 3: The Swiss Years: Writings, 1909-1911, Princeton: Princeton University Press, 1993.

[CPAE4] Klein, Martin, et al. (eds.) The Collected Papers of Albert Einstein, Vol. 4: The Swiss Years: Writings, 1912-1914, Princeton: Princeton University Press, 1995.

[CPAE5] Klein, Martin, et al. (eds.) The Collected Papers of Albert Einstein, Vol. 5: The Swiss Years: Correspondence, 1902-1914, Princeton: Princeton University Press, 1993.

[CPAE6] Kox, A.J., et al. (eds.) The Collected Papers of Albert Einstein, Vol. 6: The Berlin Years: Writings, 1914-1917, Princeton: Princeton University Press, 1996.

[CPAE8] Schulmann, Robert, et al. (eds.) The Collected Papers of Albert Einstein, Vol. 8: The Berlin Years: Correspondence, 1914-1918, Princeton: Princeton University Press, 1998.

[CPAE10] Buchwald, Diana K., et al. (eds.) The Collected Papers of Albert Einstein, Vol. 10: The Berlin Years: Correspondence, MayDecember 1920 and Supplementary Correspondence, 1909-1920, Princeton: Princeton University Press, 2006.

[Duerbeck 1987] Duerbeck, Hilmar W. "A Reference Catalogue and Atlas of Galactic Novae." Space Science Reviews 45 (1987) 1-212.

[Duerbeck 2007] Duerbeck, Hilmar W. "Novae - a Historical Perspective." In Bode, M.F., Evans, A. (eds.) Classical Novae, Cambridge University Press, forthcoming.

[Earman and Janssen 1993] Earman, John and Janssen, Michel. "Einstein's Explanation of the Motion of Mercury's Perihelion." In: Earman, John et al. (eds.) The Attraction of Gravitation, Boston: Birkhäuser, 1993 (Einstein Studies Vol. 5), 129-172. 
[Eddington 1920] Eddington, Arthur S. Space, Time, and Gravitation Cambridge: Cambridge University Press, 1920.

[Einstein 1907] Einstein, Albert. "Über das Relativitätsprinzip und die aus demselben gezogenen Folgerungen." Jahrbuch der Radioaktivität und Elektronik 4 (1907) 411-462. Reprinted in [CPAE2, Doc. 47].

[Einstein 1911] Einstein, Albert. "Über den Einfluß der Schwerkraft auf die Ausbreitung des Lichtes." Annalen der Physik 35 (1911) 898-908. Reprinted in [CPAE3, Doc. 23].

[Einstein 1912a] Einstein, Albert. "Lichtgeschindigkeit und Statik des Gravitationsfeldes." Annalen der Physik 38 (1912) 355-369. Reprinted in [CPAE4, Doc. 3].

[Einstein 1912b] Einstein, Albert. "Zur Theorie des statischen Gravitationsfeldes." Annalen der Physik 38 (1912) 443-458. Reprinted in [CPAE4, Doc. 4].

[Einstein 1912c] Einstein, Albert. "Relativität und Gravitation. Erwiderung auf eine Bemerkung von M. Abraham" Annalen der Physik 38 (1912) 1059-1064. Reprinted in [CPAE4, Doc. 8].

[Einstein 1914] Einstein, Albert. "Die formale Grundlage der allgemeinen Relativitätstheorie" Königlich Preußische Akademie der Wissenschaften (Berlin). Sitzungsberichte 1914, 1030-1085. Reprinted in [CPAE6, Doc. 9].

[Einstein 1915a] Einstein, Albert. "Zur allgemeinen Relativitätstheorie." Königlich Preußische Akademie der Wissenschaften (Berlin). Sitzungsberichte 1915, 778-786. Reprinted in [CPAE6, Doc. 21].

[Einstein 1915b] Einstein, Albert. "Zur allgemeinen Relativitätstheorie (Nachtrag)." Königlich Preußische Akademie der Wissenschaften (Berlin). Sitzungsberichte 1915, 799-801. Reprinted in [CPAE6, Doc. 22].

[Einstein 1915c] Einstein, Albert. "Erklärung der Perihelbewegung des Merkur aus der allgemeinen Relativitätstheorie (Nachtrag)." Königlich Preußische Akademie der Wissenschaften (Berlin). Sitzungsberichte 1915, 831-839. Reprinted in [CPAE6, Doc. 24]. 
[Einstein 1915d] Einstein, Albert. "Die Feldgleichungen der Gravitation." Königlich Preußische Akademie der Wissenschaften (Berlin). Sitzungsberichte 1915, 844-847. Reprinted in [CPAE6, Doc. 25].

[Einstein 1916] Einstein, Albert. "Die Grundlage der allgemeinen Relativitätstheorie." Annalen der Physik 49 (1916) 769-822. Reprinted in [CPAE6, Doc. 30].

[Einstein 1916b] Einstein, Albert. "Eine neue formale Deutung der Maxwellschen Feldgleichungen der Elektrodynamik." Königlich Preußische Akademie der Wissenschaften (Berlin). Sitzungsberichte 1916, 184188. Reprinted in [CPAE6, Doc. 27].

[Einstein and Grossmann 1913] Einstein, Albert and Grossmann, Marcel. Entwurf einer verallgemeinerten Relativitätstheorie und einer Theorie der Gravitation. Leipzig: Teubner, 1913. Reprinted in [CPAE4, Doc. 13].

[Einstein 1936] Einstein, Albert. "Lens-like action of a star by the deviation of light in the gravitational field." Science 84 (1936) 506-507.

[Fernau 1914] Fernau, Hermann. Die französische Demokratie. Sozialpolitische Studien aus Frankreichs Kulturwerkstatt. München: Duncker \& Humblot, 1914.

[Fischer-Petersen 1912] Fischer-Petersen, J. "Über die Lichtkurve der Nova (18.1912) Geminorum 2." Astronomische Nachrichten 192 (1912) 429440.

[Furuhjelm 1912] Furuhjelm, Ragnar. "Über das Spektrum der Nova Geminorum 2." Astronomische Nachrichten 192 (1912) 117-124.

[Hentschel 1994] Hentschel, Klaus. "Erwin Finlay Freundlich and Testing Einstein's Theory of Relativity." Archive for History of Exact Sciences 47 (1994) 143-201.

[Hentschel 1997] Hentschel, Klaus. The Einstein Tower. An Intertexture of Dynamic Construction, Relativity Theory, and Astronomy. Stanford: Stanford University Press, 1997.

[Janssen 1999] Janssen, Michel. "Rotation as the Nemesis of Einstein's Entwurf Theory." In: Goenner, Hubert, et al. (eds.). The Expanding Worlds 
of General Relativity Boston: Birkhäuser, 1999 (Einstein Studies Vol. 7), $127-157$.

[Janssen et al. 2007] Janssen, Michel, Norton, John D., Renn, Jürgen, Sauer, Tilman, and Stachel, John. The Genesis of General Relativity. Einstein's Zurich Notebook. Vol. 1. Introduction and Source, Vol. 2. Commentary and Essays. Dordrecht: Springer, 2007.

[Laue 1921] Laue, Max von. [Nachruf auf Emil Arnold Budde] Verhandlungen der Deutschen Physikalischen Gesellschaft (1921) 66-68.

[Lodge 1919] Lodge, Oliver J. "Gravitation and Light" Nature 104 (1919) 354.

[Ludendorff 1912] Ludendorff, H. "Über die schwachen Absorptionslinien im Spektrum der Nova Geminorum 2." Astronomische Nachrichten 192 (1912) 124-130.

[Norton 1984] Norton, John D. "How Einstein Found His Field Equations." Historical Studies in the Physical Sciences 14 (1984), 253-316.

[Norton 1992] Norton, John D. "The Physical Content of General Covariance." In: Eisenstaedt, Jean, and A.J.Kox (eds.). Studies in the History of General Relativity Boston: Birkhäuser, 1992 (Einstein Studies Vol. 3), 281-315.

[Pickering 1912] Pickering, Edward C. Astronomical Bulletin of the Harvard College Observatory 17 March 1912.

[Renn, Sauer, and Stachel 1997] Renn, Jürgen, Sauer, Tilman, and Stachel, John. "The Origin of Gravitational Lensing: A Postscript to Einstein's 1936 Science Paper." Science 275 (1997) 184-186.

[Renn and Sauer 2003] Renn, Jürgen, and Sauer, Tilman. "Eclipses of the Stars. Mandl, Einstein, and the Early History of Gravitational Lensing." In: A. Ashtekar et al. (eds.). Revisiting the Foundations of Relativistic Physics, Dordrecht: Kluwer, 2003, 69-92.

[Retter et al. 1999] Retter, A., Leibowitz, E.M., Naylor, T. "An irradiation effect in Nova DN Gem 1912 and the significance of the period gap for classical novae." Monthly Notices of the Royal Astronomical Society 308 (1999) 140-146. 
[Shara 1989] Shara, Michael M. "Recent Progress in Understanding the Eruptions of Classical Novae." Publications of the Astronomical Society of the Pacific 101 (1989) 5-31.

[Werner 1921] Werner, R. "Emil Arnold Budde." Elektrotechnische Zeitschrift 42 (1921) 1153-1154. 